\title{
甲基丙烯酸三苯甲酯不对称聚合 过程中的立体化学
}

虞 斌 林雪春 丁孟贤* 姚瑜元 王佛松

(中国科学院长佘应用化学研究所, 长春 130022)

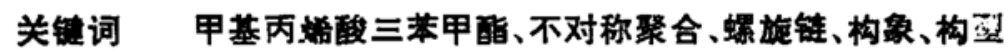

甲基丙烯酸三苯甲酯 (TrMA) 在不对称聚合中, 当有手性配体鹰爪豆碱 ((-)- $\mathrm{S}_{\mathrm{p}}$ ) 存在 时, 可形成具有单手螺旋链构象的旋光性聚合物 ${ }^{[1,2]}$. Vogt ${ }^{[3]}$ 用 TrMA 的活性二聚体作引发 剂, 聚合 TrMA, 结果表明, 右手螺旋链的聚甲基丙烯酸三苯甲酯 (PTrMA) 的主链上各不对 称碳原子均为 $\mathrm{S}$ 型, 而 Okamoto ${ }^{[4]}$ 借助色谱拆分 PTrMA 低聚物的方法得出了相反的结果。 至于手性配体决定螺旋方向的原因则未见报道. 本文从单体构象, 配体 (一) $-S_{p}$ 的构象及 PTrMA 活性端的烯醇式构型, 研究了 TrMA 在不对称聚合过程中的立体化学.

\section{一、实 验 部 分}

2-甲基丁酸三苯甲酯 ( $\operatorname{Tr} M B$ ) 由相应的酸与等摩尔的三苯氯甲烷在三乙胺催化下制得. mp. $46-48^{\circ} \mathrm{C},{ }^{1} \mathrm{H}-\mathrm{NMR}\left(\mathrm{CDCl}_{3}\right), \delta(\mathrm{ppm}): 0.76-0.90(\mathrm{t}, 3 \mathrm{H}), 1.14-1.21(\mathrm{~d}, 3 \mathrm{H}), 1.38-1.66$ $(\mathrm{m}, 2 \mathrm{H}), 2.38-2.58(\mathrm{~m}, 1 \mathrm{H}), 7.21-7.39(\mathrm{~m}, 15 \mathrm{H})$.

烯醇式 $\operatorname{Tr} M B$ 的制备: 将 $\operatorname{TrMB}$ 溶于干燥的四氢呋喃 (THF) 中, $-78^{\circ} \mathrm{C}$, 昰皆保护 下加人等摩尔的丁基锂的已烷溶液, $1 \mathrm{~h}$ 后, 加人 10 倍量的三甲基氯硅烷 $\left(\left(\mathrm{CH}_{3}\right)_{3} \mathrm{SiCl}\right)$, 反应 $30 \mathrm{~min},-20^{\circ} \mathrm{C}$ 下减压抽除 THF 和剩余 $\left(\mathrm{CH}_{3}\right)_{3} \mathrm{SiCl}$. ${ }^{1} \mathrm{H}-\mathrm{NMR}$ 用 Unity 400 核磁共振仪 记录. 化合物最稳定构象能用 Allinger 分子力学 MMP 2 程序计算.

\section{二、结果与讨论}

1. 单体 TrMA 的构象对于 $\alpha 、 \beta$ 不饱和酯或酮, $\mathrm{C}=\mathrm{C}$ 与 $\mathrm{C}=\mathrm{O}$ 之间有 $\mathrm{s}$ 顺和 $\mathrm{s}$ 反两种构象. 四圆衍射表明, 固态 TrMA 呈 $s$-顺式构象 ${ }^{[}$, 其在溶液中的构象, 则可借助

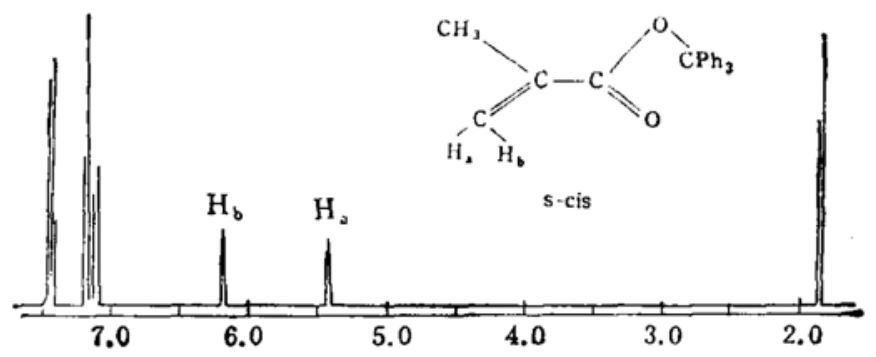

图 1 TrMA 的 'H-NMR 谱图(溶剂: $\mathrm{C}_{6} \mathrm{D}_{4} \mathrm{Cl}_{2}$ )

1992-01-20 收稿, 1992-04-07 收修改稿

* 遮㴖联系人

第 22 期

科学通报

205 r 
: H-NMR 来研究. 众所周知, 在 $s$-顺式 $\alpha 、 \beta$ 不饱和酯或酮中, $\mathrm{C}=\mathrm{O}$ 的去屏蔽作用使得 $\beta$ 碳 原子上的两个质子的化学位移的差变大, 尤其在芳烃溶剂中. 对于 TrMA, 这个差值为 0.762 $\mathrm{ppm}$ （图 1), 与仅存在 $s$-顺式构象的 $\alpha$-亚甲基戊内酯的值 $\left(0.83 \mathrm{ppm}\right.$ )相近 ${ }^{[0]}$, 而比 $s$-反式的 $\alpha 、 \beta$ 不饱和酮的 $0.19 \mathrm{ppm}$ 大得多 ${ }^{[n}$, 因而, 溶液中的 $\operatorname{TrMA}$ 也以 $\mathrm{s}$-顺式构象存在.

2. 手性配体(一)-Sp 的构象 (一)-Sp在与金属离子配位后, 呈全椅式构象. X 衍射 表明, 其形成的手性空间的右前方较左后方大 ${ }^{[8]}$, 俯视其分子模型可见, 这个空间从大到小的 走向为顺时针方向如图 2 所示.

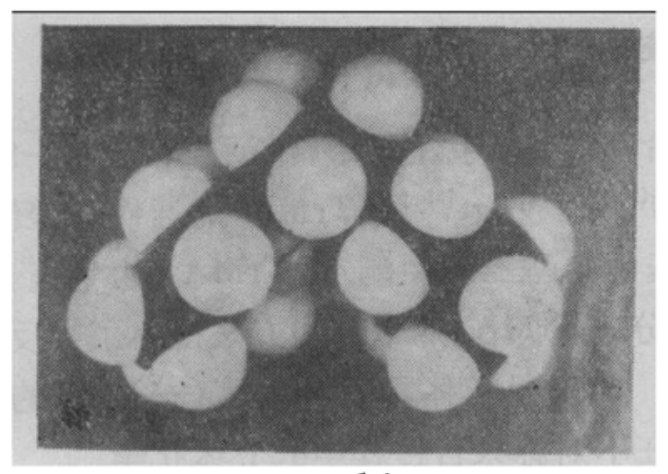

(a)

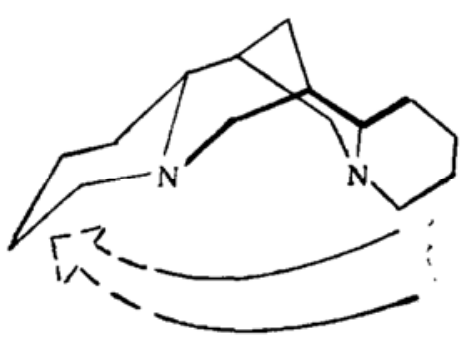

(b)

图 2 (-) $-S_{p}$ 的分子模型 ( a ) 及其手性空间走向 (b)

3. PTrMA 活性末端构型 PTrMA 活性端有两种可能的烯醇式构 型: $\mathbf{Z}$ 型和 $\mathbf{E}$ 型

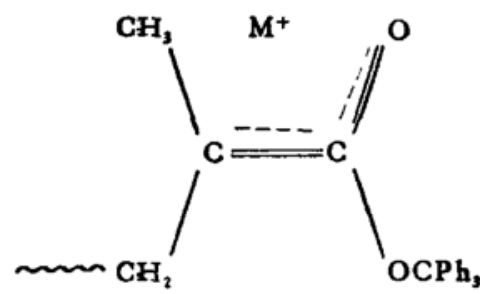

E

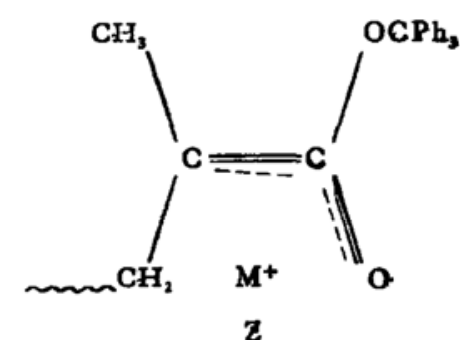

$\mathbf{z}$

${ }^{{ }^{n} 3} \mathrm{C}-\mathrm{NMR}$ 研究表明, 聚甲基丙烯酸甲酯的活性端烯醇式构型为 $Z$ 型回, 对于 PTrMA, 由于其 溶解性差, 测试时易析出, 而难以记录 ${ }^{13} \mathrm{C}-\mathrm{NMR}$ 谱, 但用分子力学计算相应两种构型的模型化
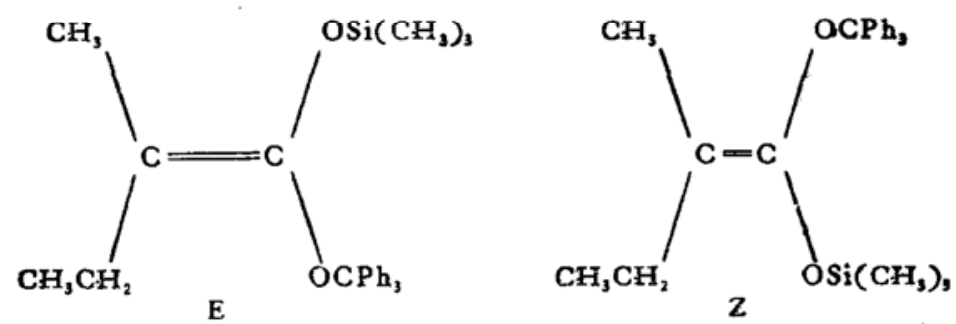

合物的最稳定构象能表明, $\mathrm{Z}$ 型构象能为 $1387.0407 \mathrm{~kJ}$ 。而 $\mathrm{E}$ 型则为 $1421.4194 \mathrm{~kJ}, \mathrm{Z}$ 型可尽量 减小末端 $\mathrm{Ph}_{3} \mathrm{C}$ 基团与倒数第二、第三单元的庞大的空间位阻.

4. PTrMA 蝶旋方向的确定 在 TrMA 的不对称聚合中, 配体(一)- $S_{\mathrm{p}}$ 始终存在于活 性末端, TrMA 是络合后再插人到分子链中. 如前所述, (一) $-S_{\mathrm{p}}$ 为聚合形成了一顺时针方向 的手性空间, 已形成的聚合物链空间体积大, 自然要占据这个空间较大的一端, 单体只能从 $\mathrm{S}_{\mathbf{p}}$ 下面的左后方接近活性中心: 

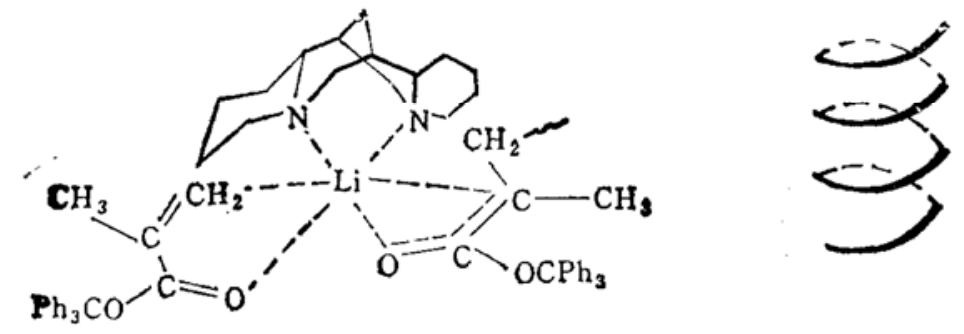

当其络合插人后, 另一单体也在接近活性中心, 并把新形成的链节单元推送到 $S_{\mathrm{p}}$ 的右前方, 也 就是说, 分子链按配体形成的手性空间走向增长, 作为结果, 右手螺旋链的 PTrMA 形成了. 而试图反方向增长的活性中心, 即单体从右前方接近活性端, 势必会造成聚合物链被挤到配体 形成的手性空间较小的一端, 尽管此时单体络合时位阻减小, 但插人时位阻大大增加, 分子链 继续增长大为困难, 这也就解释了本条件下的左手螺旋链的 PTrMA 仅停留在低聚体阶段 ${ }^{[10]}$.

5. 右手螺旋链 PTrMA 中手性碳原子绝对构型 TrMA 分子中具有庞大的 $\mathrm{Ph}_{3} \mathrm{C}$ 基 团, 无论是活性末端, 还是被络合的单体, $\mathrm{Ph}_{3} \mathrm{C}$ 总是要尽量远离 $\mathrm{S}_{\mathrm{p}}$ 的刚性骨架, 且同时满足 单体的构象 ( $s$ 顺) 和活性端构型 (Z型), 因而在聚合时, 只能形成上图所示的立体配置, 也就 是说, 单体从 $\mathrm{Si}$ 面接近活性中心 (为简便起见, 省去单体与 $\mathrm{Li}$ 的络合与脱络过程):

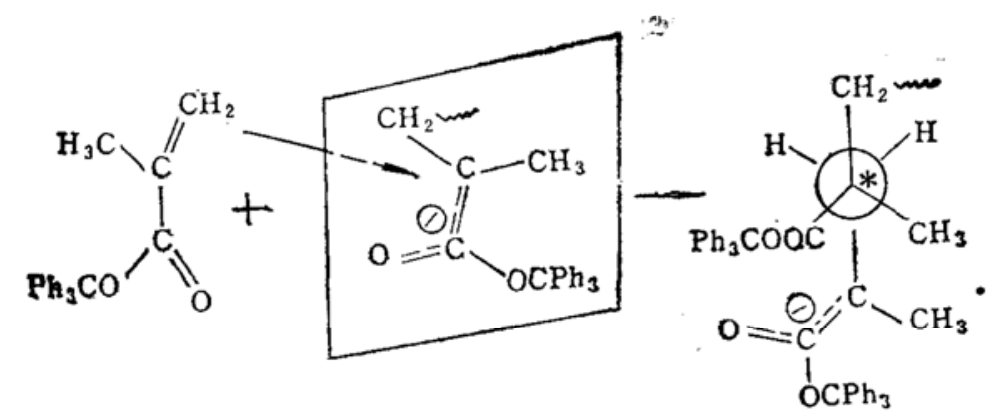

显而易见, 倒数第二单元中新生成的不对称碳原子的绝对构型为 $\mathrm{S}$ 型, 末端构型仍为 $\mathrm{Z}$ 型, 随 链的不断增长, 最终形成了---SSS- - 的全同螺旋链. 在中止反应中, 由于氢原子体积太小, 因 而立体选择性差,两和构型的几率相近. 至此我们得出结论, 手性配体 $S_{p}$ 形成的顺时针方何 的手性空间, 单体 TrMA 的 s-顺式构象及活性端的 Z 型结构, 共同决定了本条件下形成的旋 光性 PTrMA 为---SSS---全同的右手螺旋链聚合物.

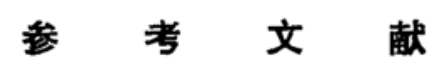

[1] Okamoto, Y. et al., J. Am. Chem. Soc, 101(1979), 4763.

[2] 王跃川等,高分子学报, 1988,4: 317 .

[3] Vogt, B., Wulff, G., Polym. Preprints, 30(1989), 2:406.

[ 4 ] Okamoto, Y., Yashima, E., Prog. Polym. Sci., 15(1990), 263.

[5] Kageyama, H. et al, Makromol. Chem., 186(1985), 203.

[6] Harmon, A. D., Hutchinsen, C. R., Tetrahedron Lett., 1973, 1293.

[ 7 ] Naito, I., Kinoshita, A. et 2l., Buil. Chem. Soc. Jpn., Jpn., 49(1976), 339.

[ 8 ] Okamoto, Y. et al., J. Am. Chem. Soc, 104(1982), 4618.

[9] Brittain, W., Polym. Preprints, 29(1998), 2:312.

[10] Okamoto, Y. et al., Chem. Lett., 1987, 759.

第 22 期

科学通报

2053 